\title{
Supporting Information \\ Ultrapure Blue Phosphorescent Organic Light-Emitting Diodes Employing a Twisted Pt(II) Complex
}

Yumeng Chen, Chunyue Qian, Ke Qin, Hongbo Li, Xiaobo Shi, Zhenzhong Lu*, Huili Ma, Tianshi Qin, Xiao-Chun Hang*, and Wei Huang*

E-mail for Prof. Z. Lu, Prof. X. Hang, Prof. W. Huang: iamzzlu@njtech.edu.cn; iamxchhang@njtech.edu.cn; iamwhuang@njtech.edu.cn

Address for Y. Chen, C. Qian, K. Qin, Prof. Z. Lu, Prof. H. Ma, Prof. T. Qin, Prof. X.-C. Hang, Prof. W. Huang. Key Laboratory of Flexible Electronics (KLOFE) \& Institute of Advanced Materials (IAM), Nanjing Tech University (NanjingTech), 30 South Puzhu Road, Nanjing 211816, China

Address and E-mail for Dr. H. Li: lihongbo.bjhy@sinopec.com, Yanshan branch of Beijing Research Institute of Chemical Industry, Sinopec, Beijing 102500, China

Address and E-mail for Dr. X. Shi, E-mail: shixb@jitriioo.com, Jiangsu JITRI Organic Optoelectronics Technology Co., Ltd., 1198 Fenhu Dadao, Wujiang, Suzhou, Jiangsu 215215, China

The $2^{\text {nd }}$ address for Prof. W. Huang, Frontiers Science Center for Flexible Electronics (FSCFE) \& Shaanxi Institute of Flexible Electronics (SIFE), Northwestern Polytechnical University (NPU), 127 West Youyi Road, Xi'an 710072, China. 


\section{Synthesis and Structural Characterization}

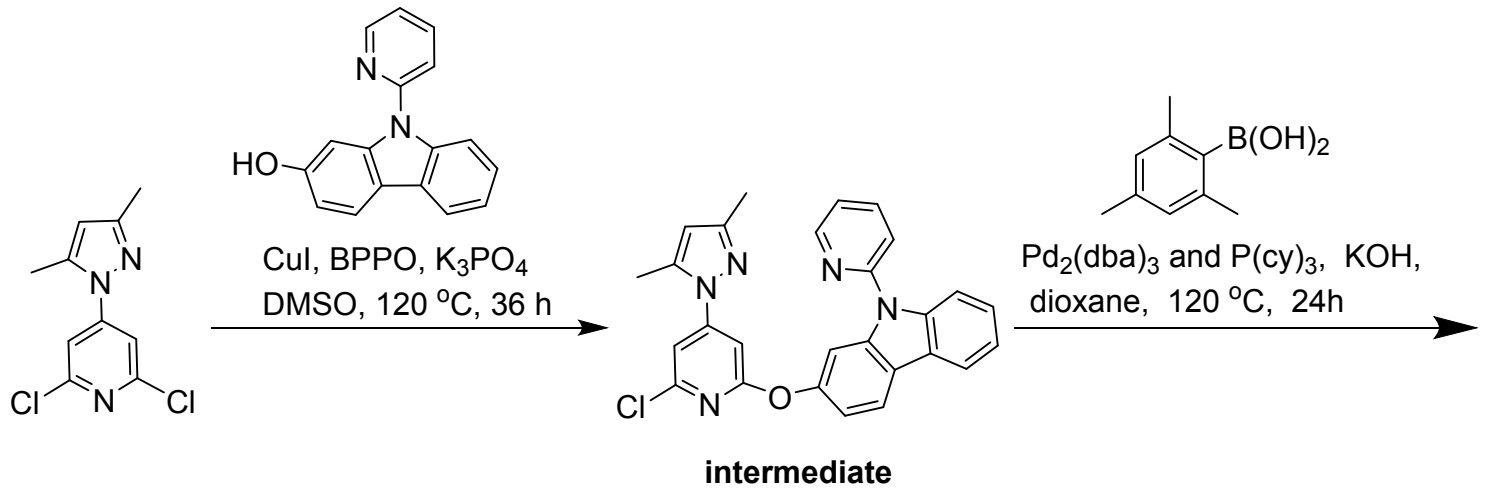

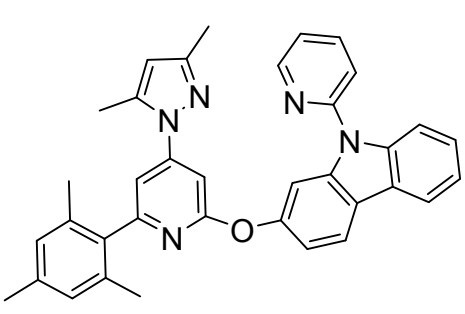

Ligand precursor

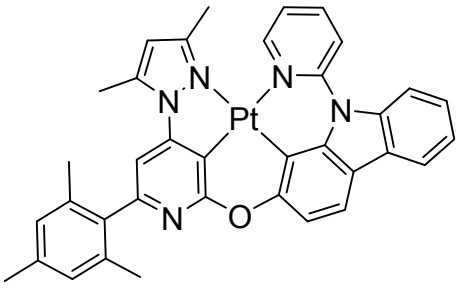

Pt(mpzpOczpy-mesi)

Figure S1. Synthesis of Platinum complexes 


\section{X-Ray Crystallography Data}

Table S1. Crystallographic data of Pt(mpzpyOczpy-mesi).

\begin{tabular}{|c|c|}
\hline Categories & Pt(mpzpyOczpy-mesi) \\
\hline Empirical formula & $\mathrm{C}_{34} \mathrm{H}_{25} \mathrm{~N}_{5} \mathrm{OPt}$ \\
\hline Formula weight & 714.17 \\
\hline Crystal system & Monoclinic \\
\hline $\mathrm{Pt} 1-\mathrm{C} 5 / \AA$ & $1.948(7)$ \\
\hline $\mathrm{Pt} 1-\mathrm{C} 2 / \AA$ & $1.969(7)$ \\
\hline Pt1-N1 / Å & $2.092(6)$ \\
\hline Pt1-N4/ $\AA$ & $2.115(6)$ \\
\hline $\mathrm{C} 5-\mathrm{Pt} 1-\mathrm{C} 2 /^{\circ}$ & $91.0(3)$ \\
\hline $\mathrm{C} 5-\mathrm{Pt} 1-\mathrm{N} 1 /^{\circ}$ & $166.7(3)$ \\
\hline C2-Pt1-N1 / ${ }^{\circ}$ & $79.8(3)$ \\
\hline $\mathrm{C} 5-\mathrm{Pt} 1-\mathrm{N} 4 /^{\circ}$ & $90.7(3)$ \\
\hline $\mathrm{C} 2-\mathrm{Pt} 1-\mathrm{N} 4 /^{\circ}$ & $165.4(3)$ \\
\hline N1-Pt1-N4 / ${ }^{\circ}$ & $100.6(2)$ \\
\hline Pz-Py1/ ${ }^{\circ}$ & 57.24 \\
\hline $\mathrm{Pz}-\mathrm{Py} 2 /^{\circ}$ & 15.95 \\
\hline
\end{tabular}

Table S2. Crystallographic data of Pt(pzpyOczpy- $i \operatorname{Pr})$.

\begin{tabular}{ll}
\hline Categories & Pt(pzpyOczpy-iPr) \\
\hline Pz-Py1 ${ }^{\circ}$ & 41.01 \\
Pz-Py2 ${ }^{\circ}$ & 1.91 \\
\hline
\end{tabular}




\section{Computational Simulations}
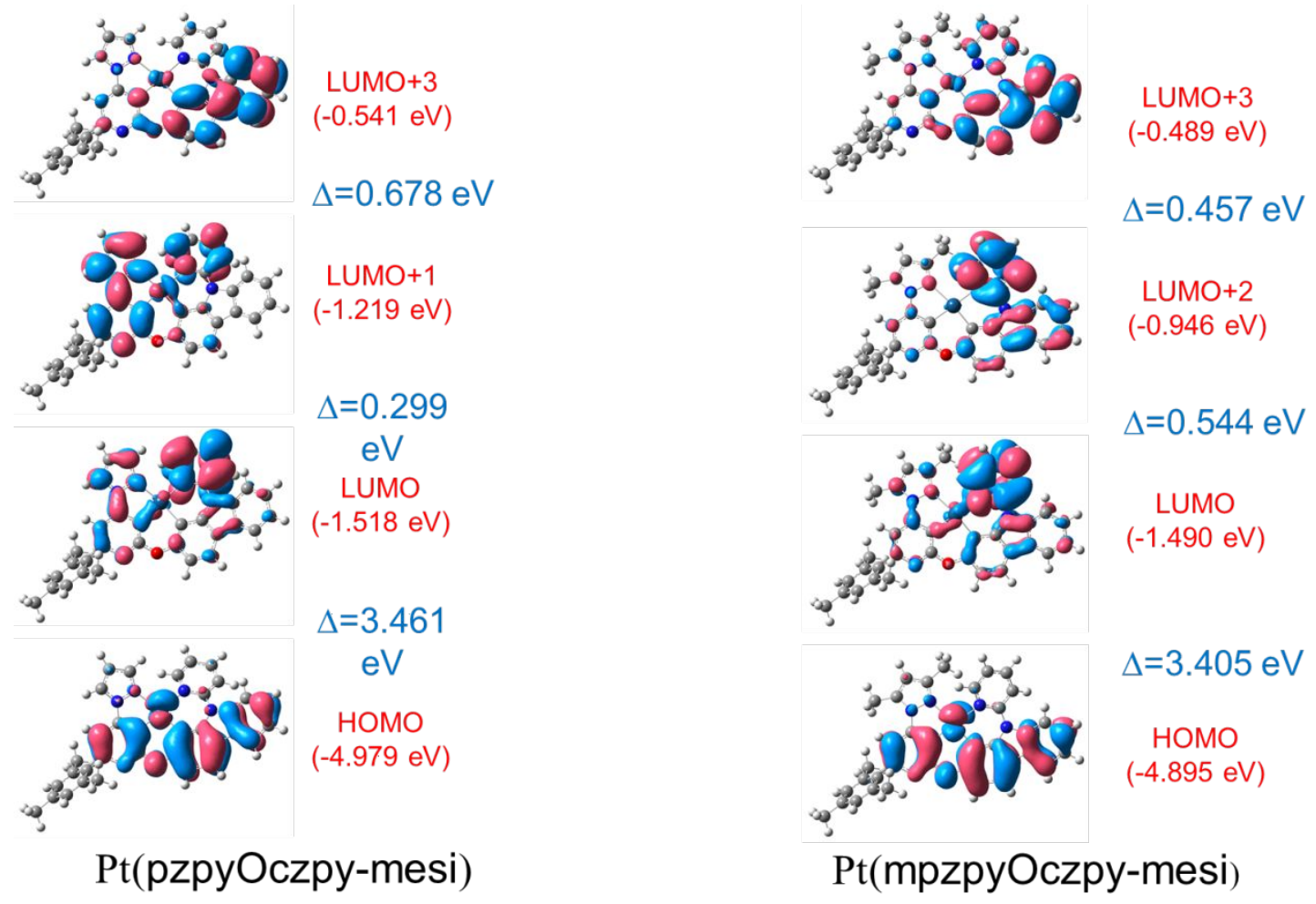

Figure S2. Frontier molecular orbitals calculated by using (TD)B3LYP functional, together mixed basis set (6-31G* for C H O N; lanl2dz for Pt) 
Thermal stability

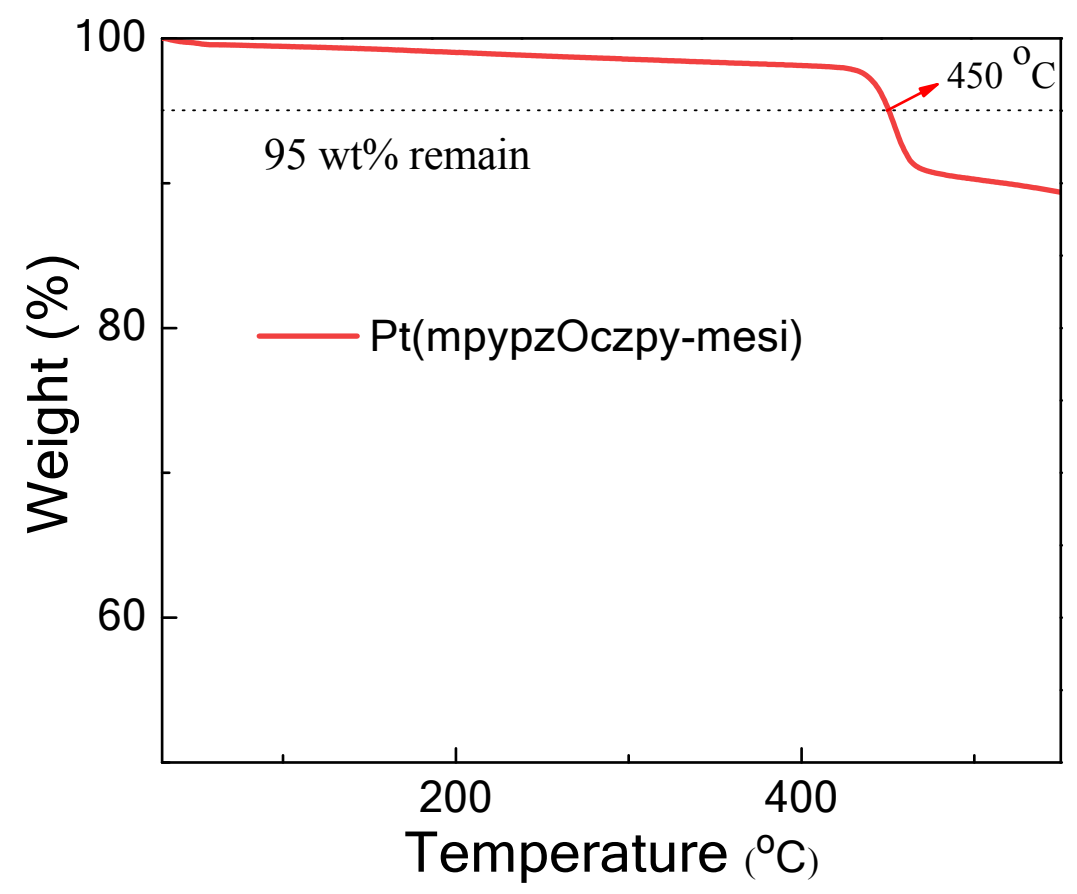

Figure S3 Thermal gravimetric analysis.

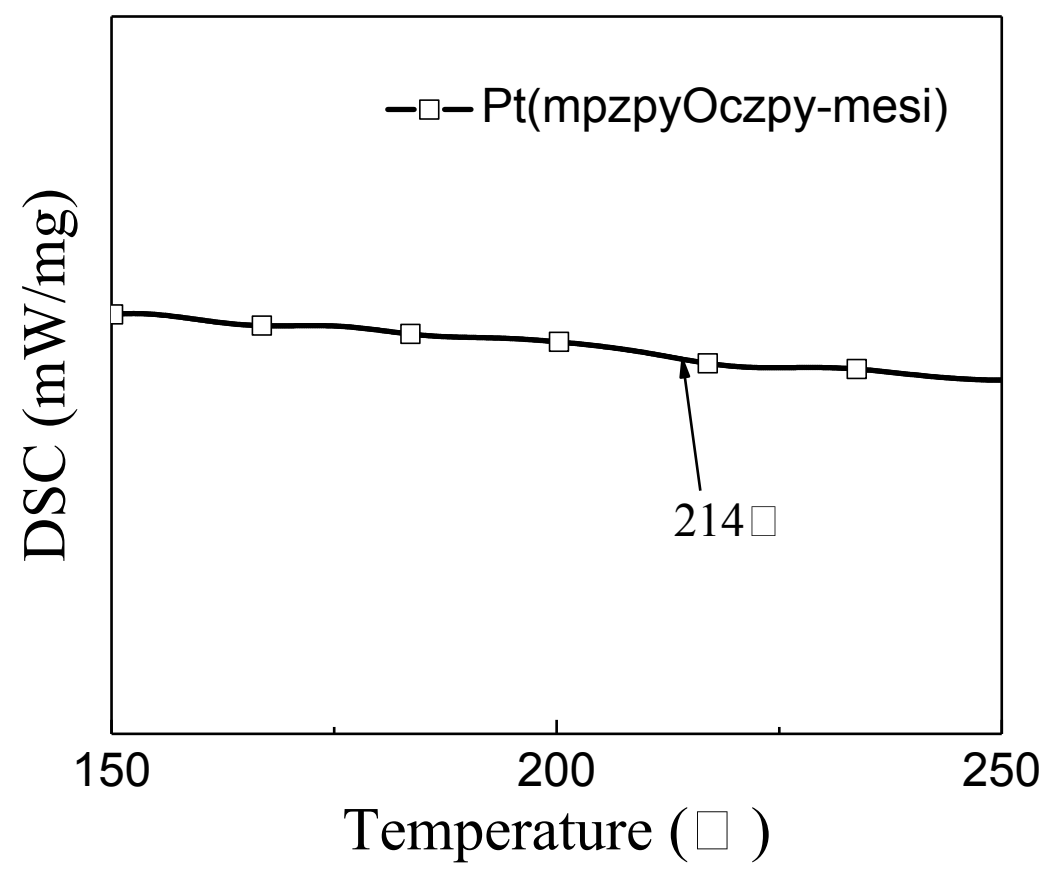

Figure S4 Differential scanning calorimetry analysis. 


\section{Photostability}

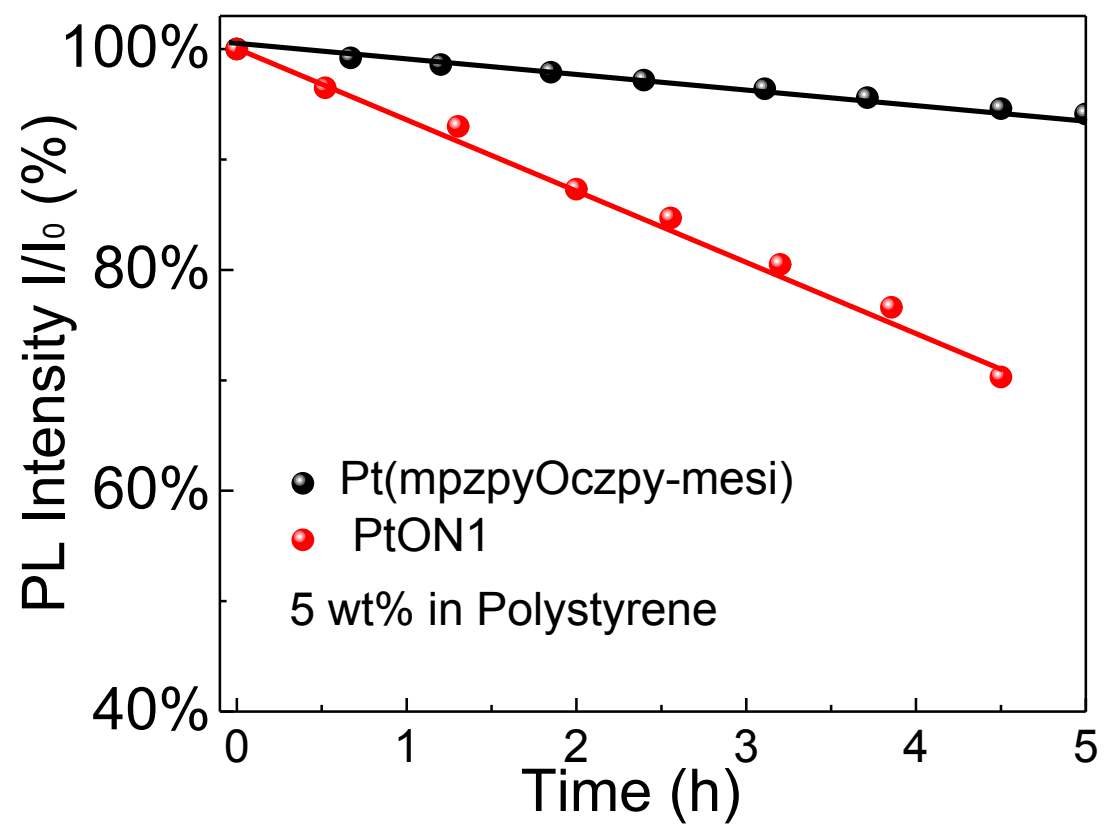

Figure S5. Photodegradation curves. 


\section{Photophysical Properties}

Table S3. Concentration depended photoluminescent data of Pt(mpzpyOczpy-mesi).

\begin{tabular}{cccc}
\hline Doping Concentration & PLQY & $K_{\mathrm{r}}\left[10^{5} \mathrm{~s}^{-1}\right]$ & $K_{\mathrm{nr}}\left[10^{5} \mathrm{~s}^{-1}\right]$ \\
\hline $1 \%$ & $98 \%$ & 2.6 & 0.05 \\
$5 \%$ & $68 \%$ & 2.3 & 1.06 \\
$10 \%$ & $55 \%$ & 2.0 & 1.63 \\
$30 \%$ & $21 \%$ & 1.0 & 3.76 \\
$50 \%$ & $14 \%$ & 0.8 & 4.65 \\
\hline
\end{tabular}

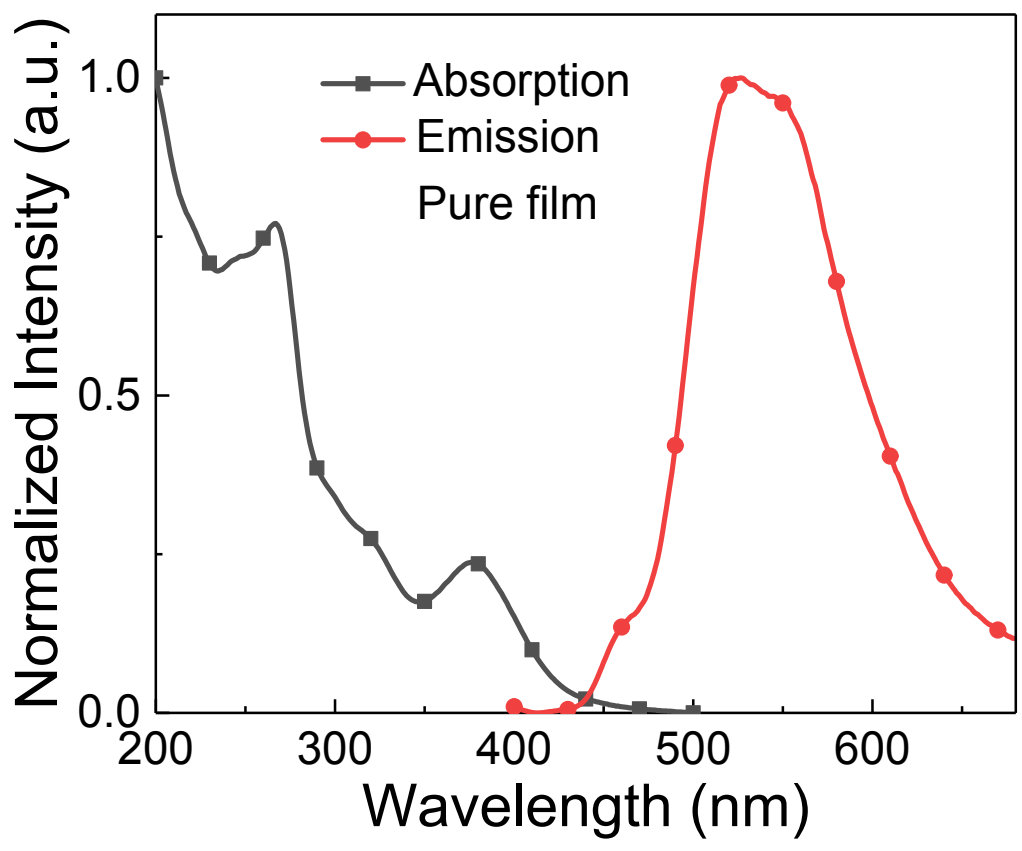

Figure S6. Emission and absorption of amorphous film. 


\section{Device performances}

(a)

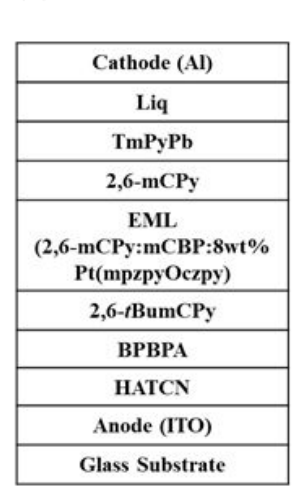

(b)

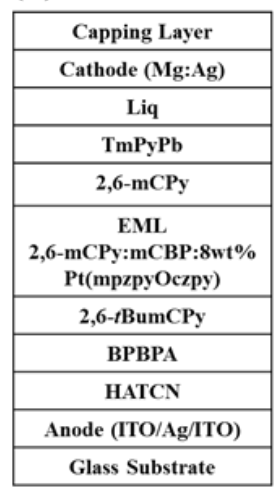

(c)

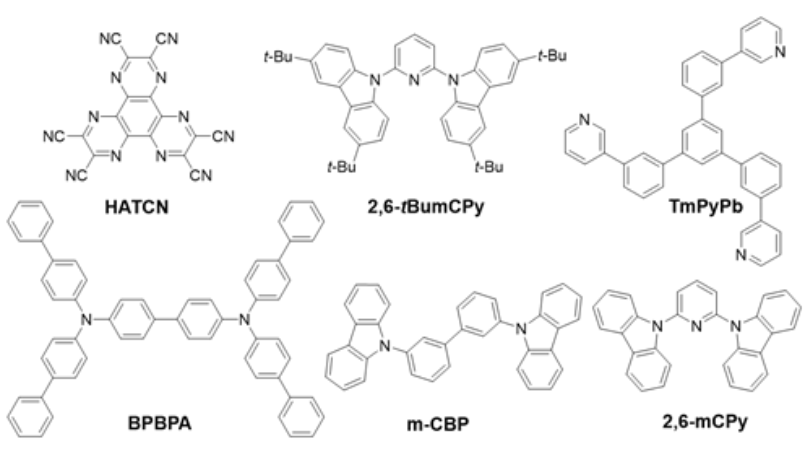

Figure S7. Device structures of BE-PHOLED (a), TE-PHOLED (b) and functional molecules (c).

(a)

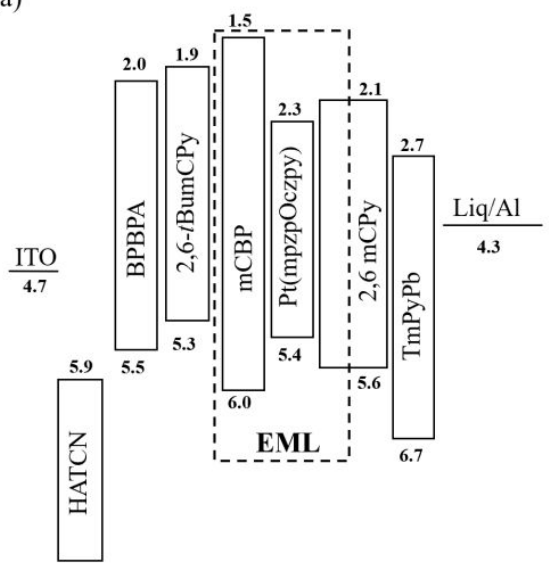

(b)

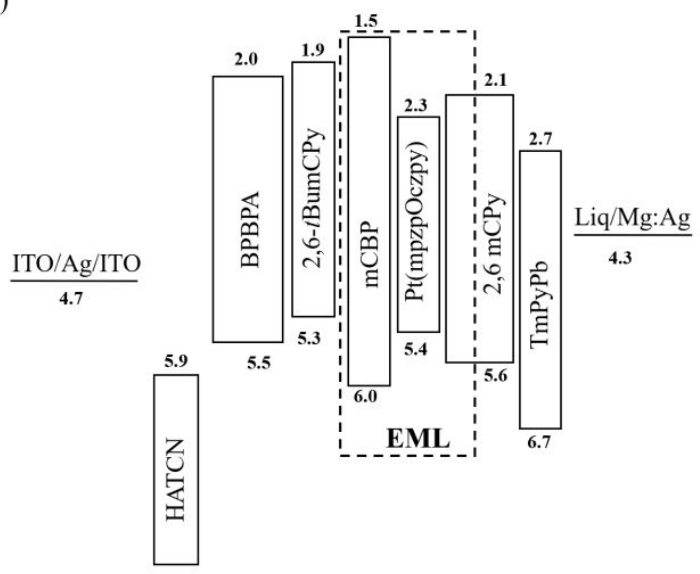

Figure S8. Energy levels of BE PHOLED (a) and TE PHOLED (b).

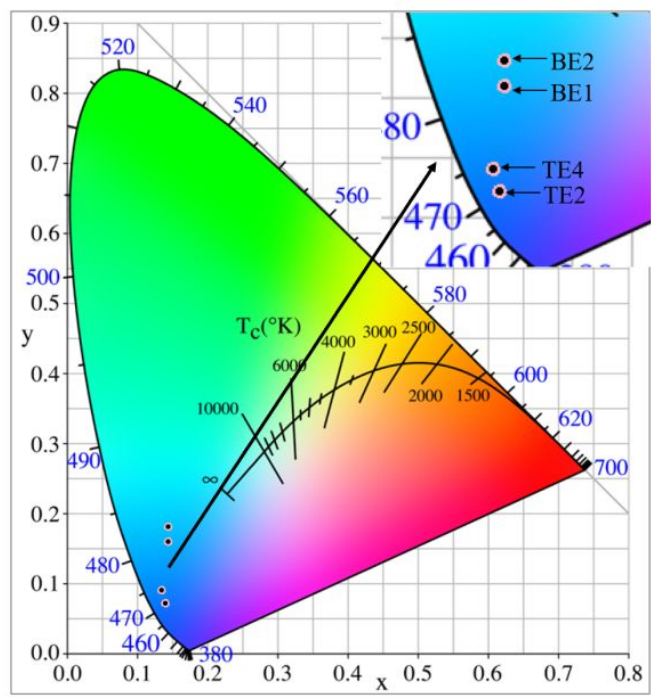

Figure S9 CIE diagram of the devices BE1, BE2, TE2 and TE4. 


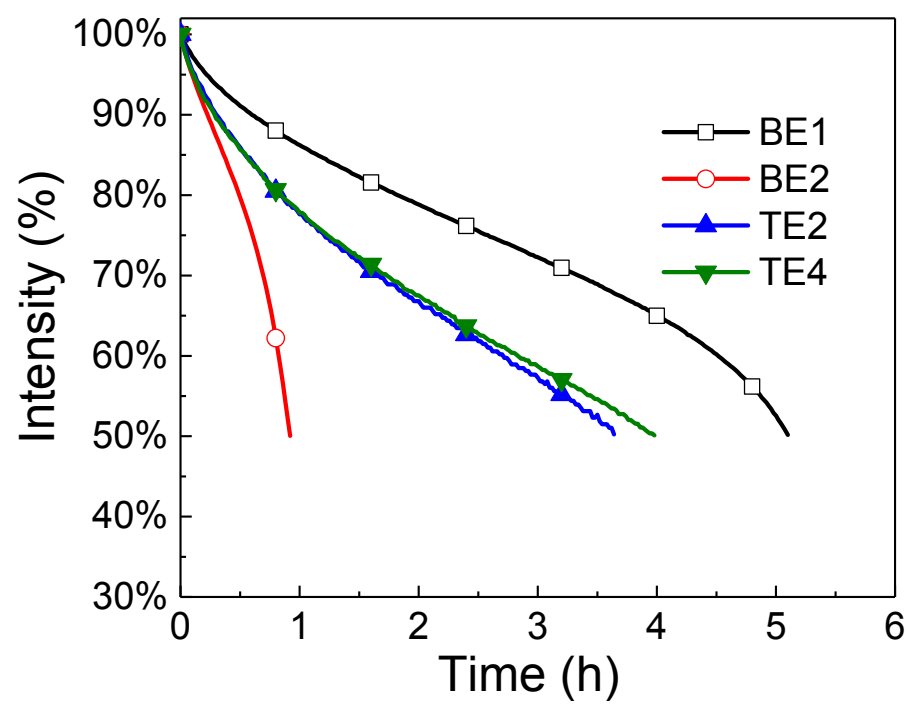

Figure S10 Lifetimes of BE1, BE2, TE2 and TE4.
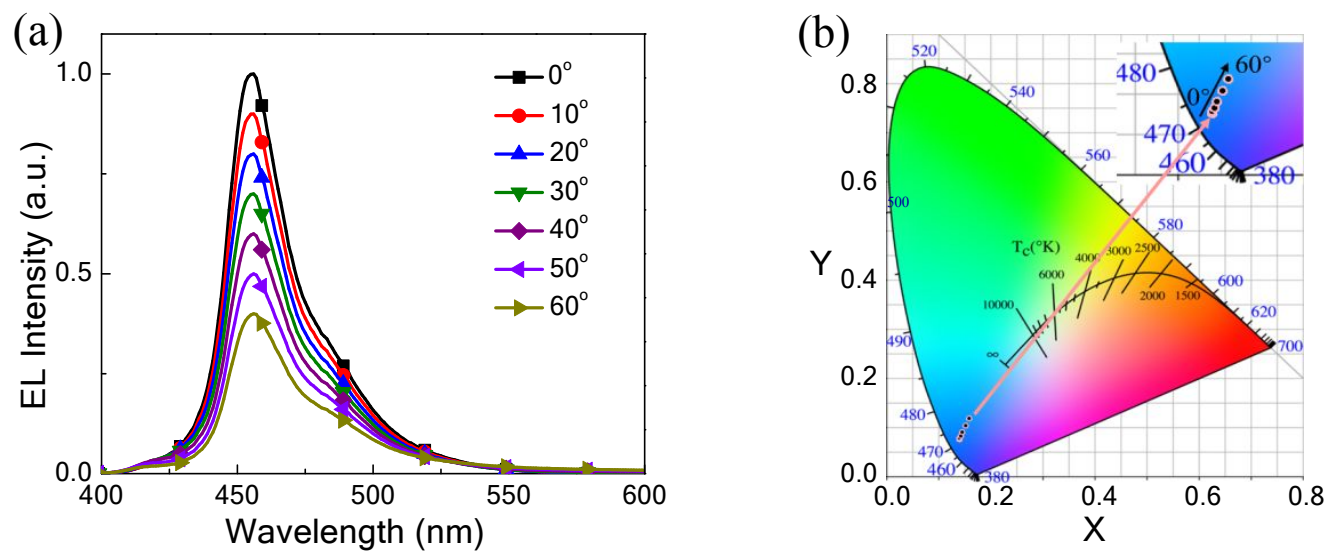

Figure S11 Angular distributions of TE2. (a) EL spectrum; (b) Color coordinates.

Table S4. Device Performances of PtON1

\begin{tabular}{|c|c|c|c|c|}
\hline Device $^{\text {a }}$ & $\begin{array}{l}\mathbf{x}, \mathbf{y}^{\mathbf{b}} \\
{[\mathbf{n m}]}\end{array}$ & $\begin{array}{c}\text { Peak, FWHM, L, CIE }(x, y)^{c} \\
{\left[\mathrm{~nm}, \mathrm{~nm}, \mathrm{~cd} \mathrm{~m}^{-2},-\right]}\end{array}$ & $\begin{array}{c}\text { EQE, PE, CE d } \\
{\left[\%, \operatorname{lm~} \mathbf{W}^{-1},{\left.\operatorname{cd~} A^{-1}\right]}^{[\%}\right.}\end{array}$ & 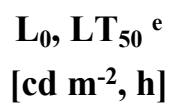 \\
\hline BE5 & 20,30 & $461,63,5926,(0.145,0.194)$ & $9.6,6.4,14.0$ & $646,2.44$ \\
\hline BE6 & 30,30 & $464,63,5920,(0.146,0.205)$ & $9.4,6.5,14.2$ & $685,2.18$ \\
\hline
\end{tabular}

${ }^{a}$ BE5 6 are in the structure of ITO/HATCN $(10 \mathrm{~nm}) /$ BPBPA $(70 \mathrm{~nm}) / 2,6-t$ BumCPy $(10 \mathrm{~nm}) / 2,6 \mathrm{mCPy}: \mathrm{mCBP}:$ PtON1 (46\%:46\%:8\%, x nm)/26mcpy $(10 \mathrm{~nm}) / \mathrm{TmPyPb}(\mathrm{y} \mathrm{nm}) / \mathrm{Liq} / \mathrm{Al}(120 \mathrm{~nm}) .{ }^{\mathrm{b}} \mathrm{x}, \mathrm{y}$ are the thickness of correlated functional layers. ${ }^{\mathrm{c}}$ The value was recorded at $50 \mathrm{~mA} \mathrm{~m}^{-2}$ at $0^{\circ}$ viewing angle in the front. ${ }^{\mathrm{d}}$ The values of current efficiency (CE), power efficiency (PE) and external quantum efficiency (EQE) were recorded at a luminance of $1000 \mathrm{~cd} \mathrm{~m}^{-2}$. ${ }^{\mathrm{e}}$ LT50 lifetimes at initial luminance of $\mathrm{L}_{0}$ were measured under a constant current density of $4 \mathrm{~mA} \mathrm{~m}^{-1}$. 


\section{${ }^{1} \mathrm{H}$ NMR and ${ }^{13} \mathrm{C}$ NMR Spectra}

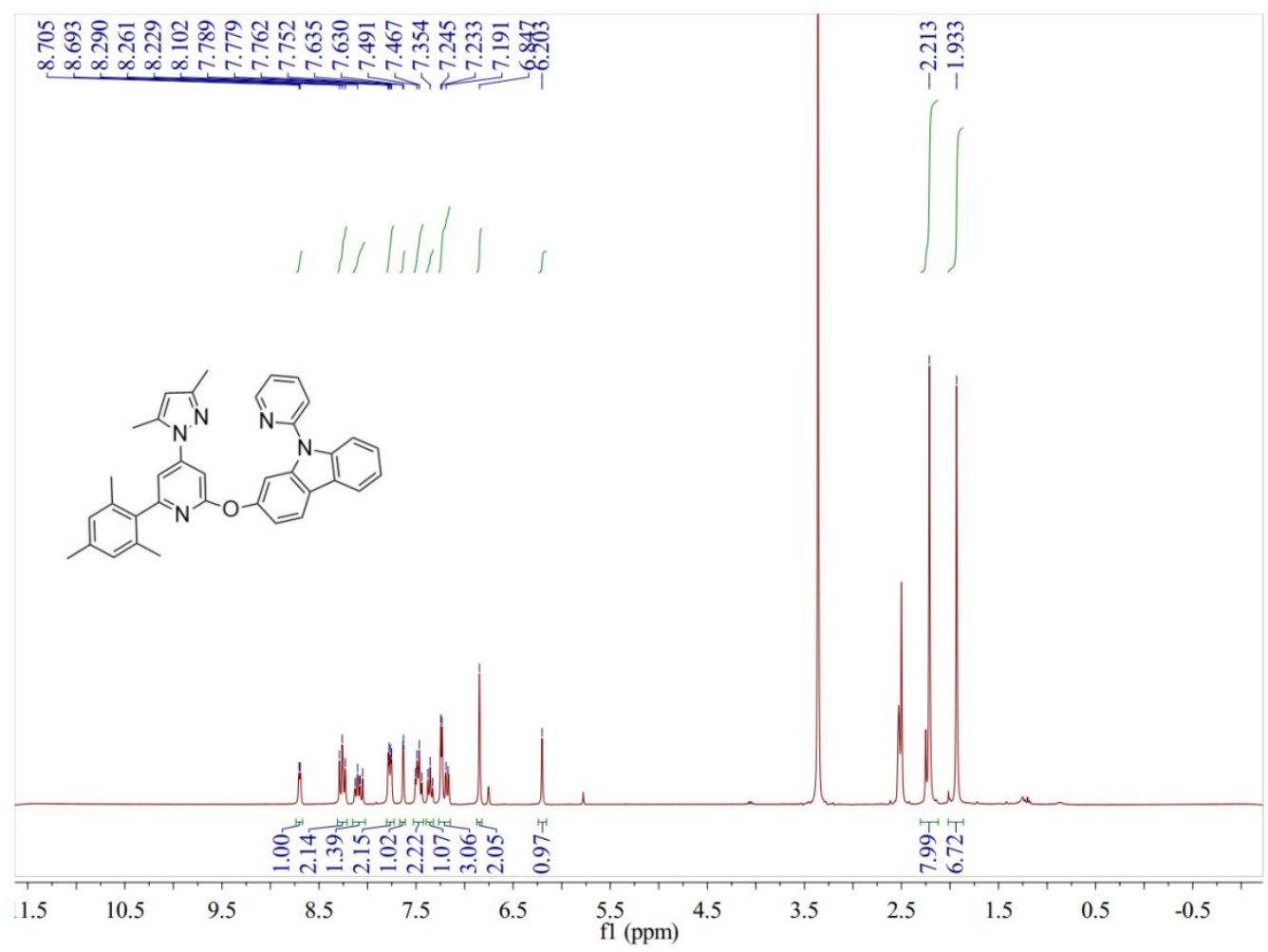

Figure S12 ${ }^{1} \mathrm{H}$ NMR of Ligand $\mathbf{b}$

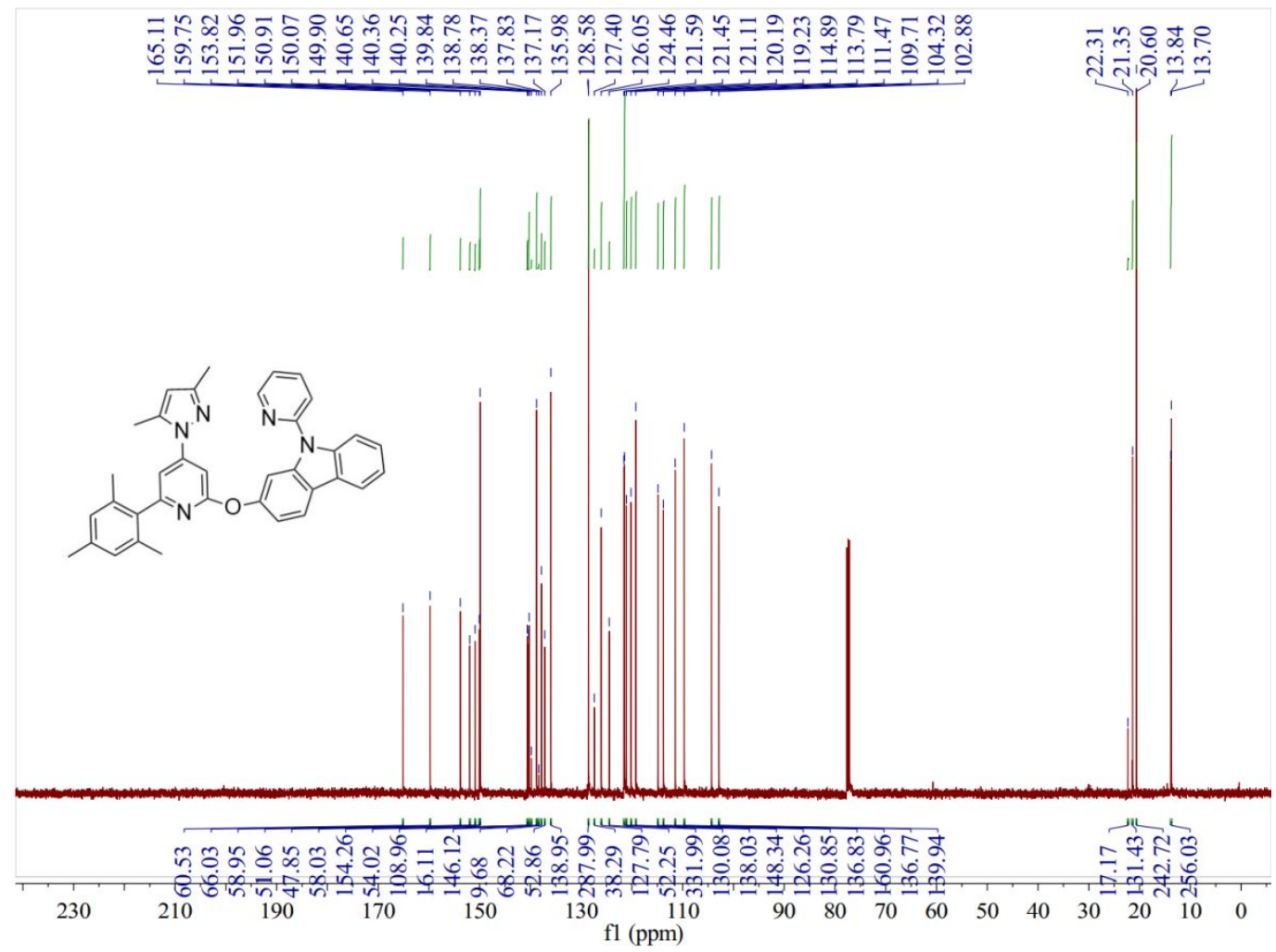

Figure S13 ${ }^{13} \mathrm{C}$ NMR of Ligand Precursor $b$ 


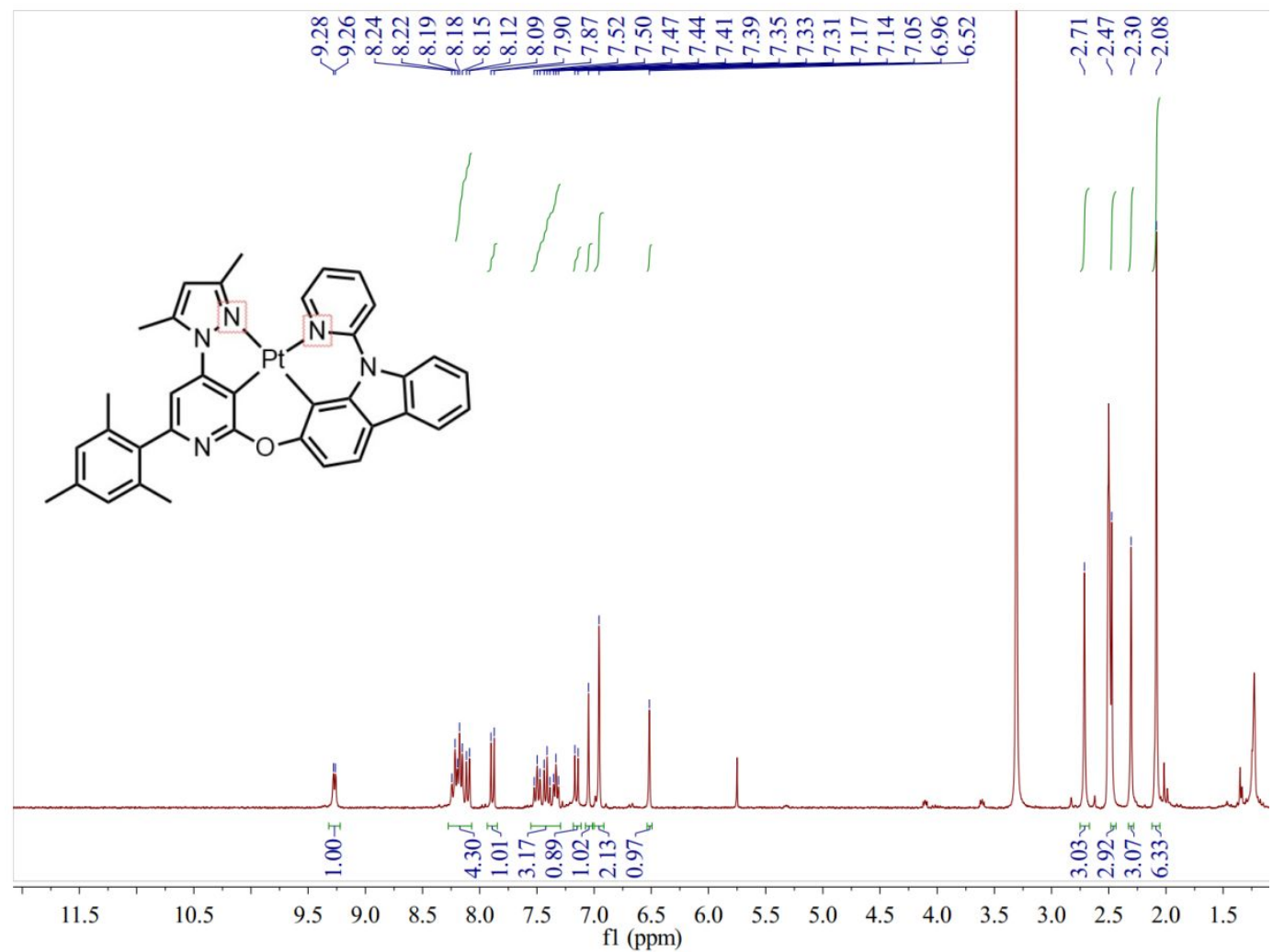

Figure S14 ${ }^{1} \mathrm{H}$ NMR of Pt(mpzpyOczpy-mesi)

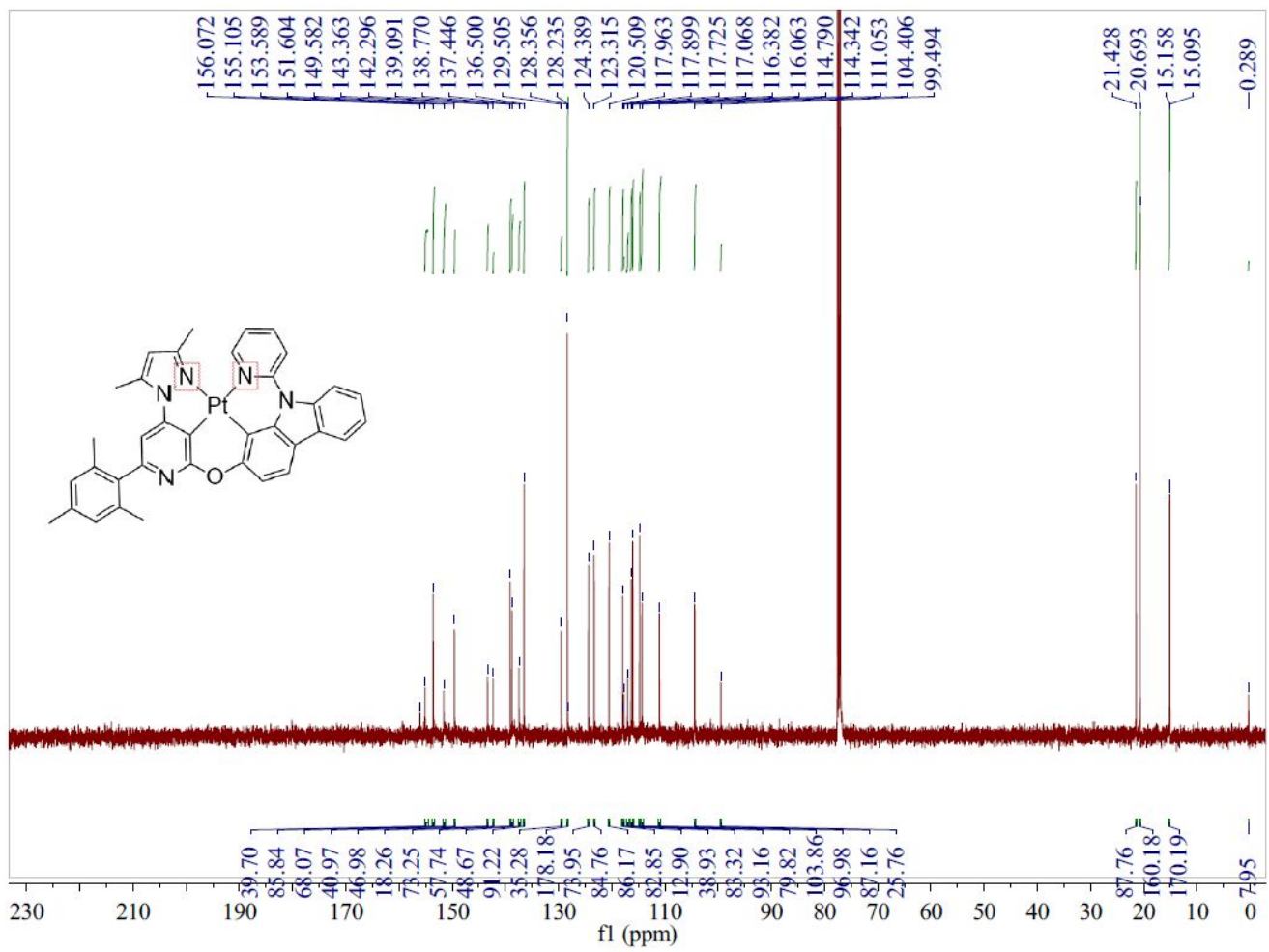

Figure S15 ${ }^{13} \mathrm{C}$ NMR of Pt(mpzpyOczpy-mesi) 


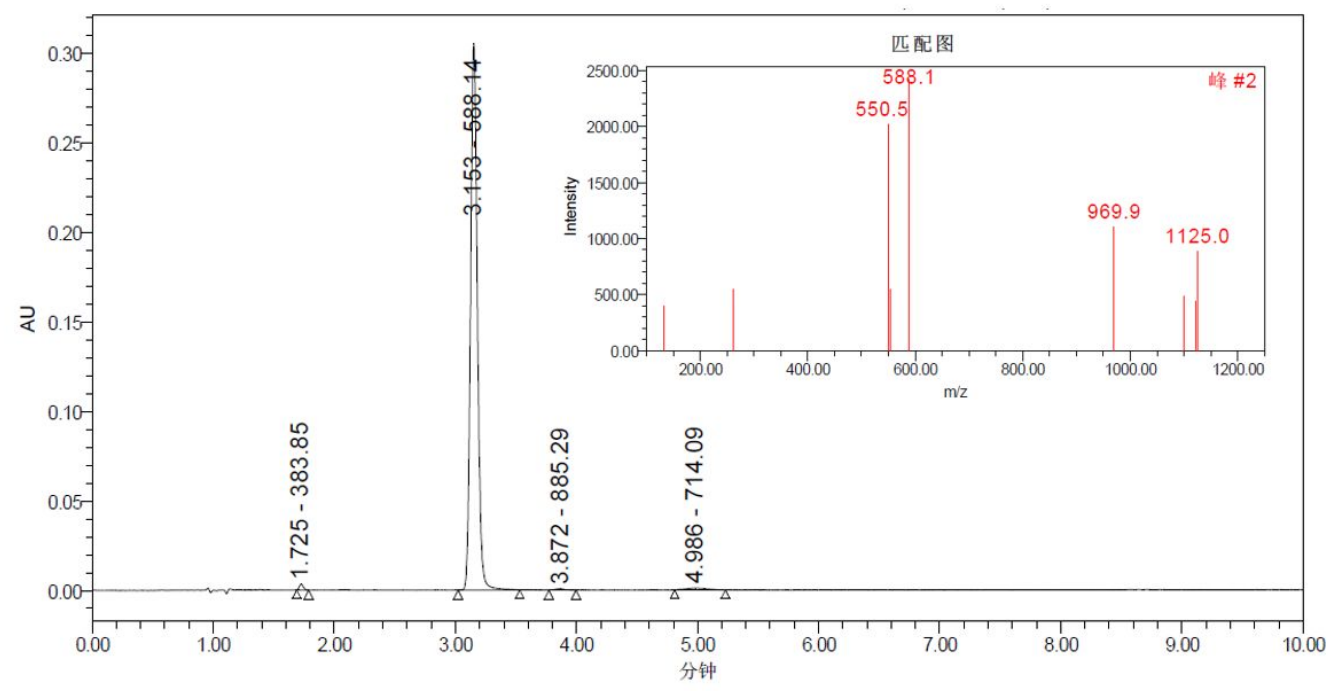

Figure S16 LC-Mass spectroscopy of Ligand Precursor

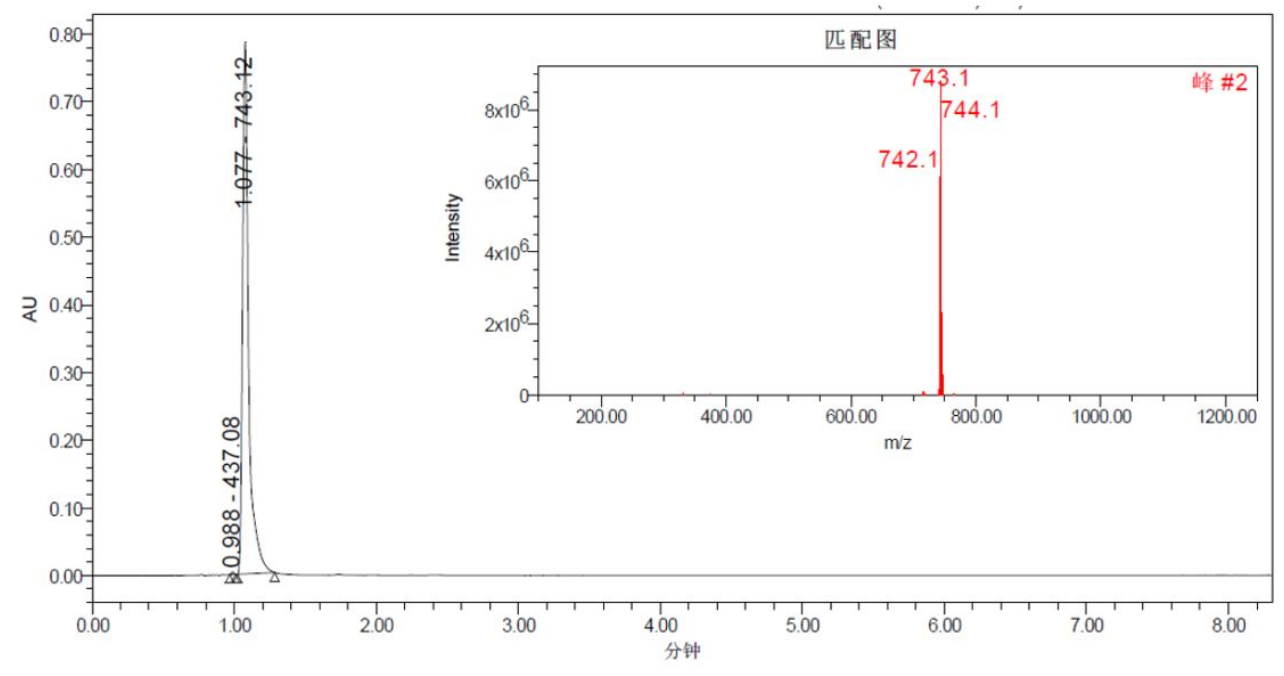

Figure S17 LC-Mass spectroscopy of Pt(mpzpyOczpy-mesi) 

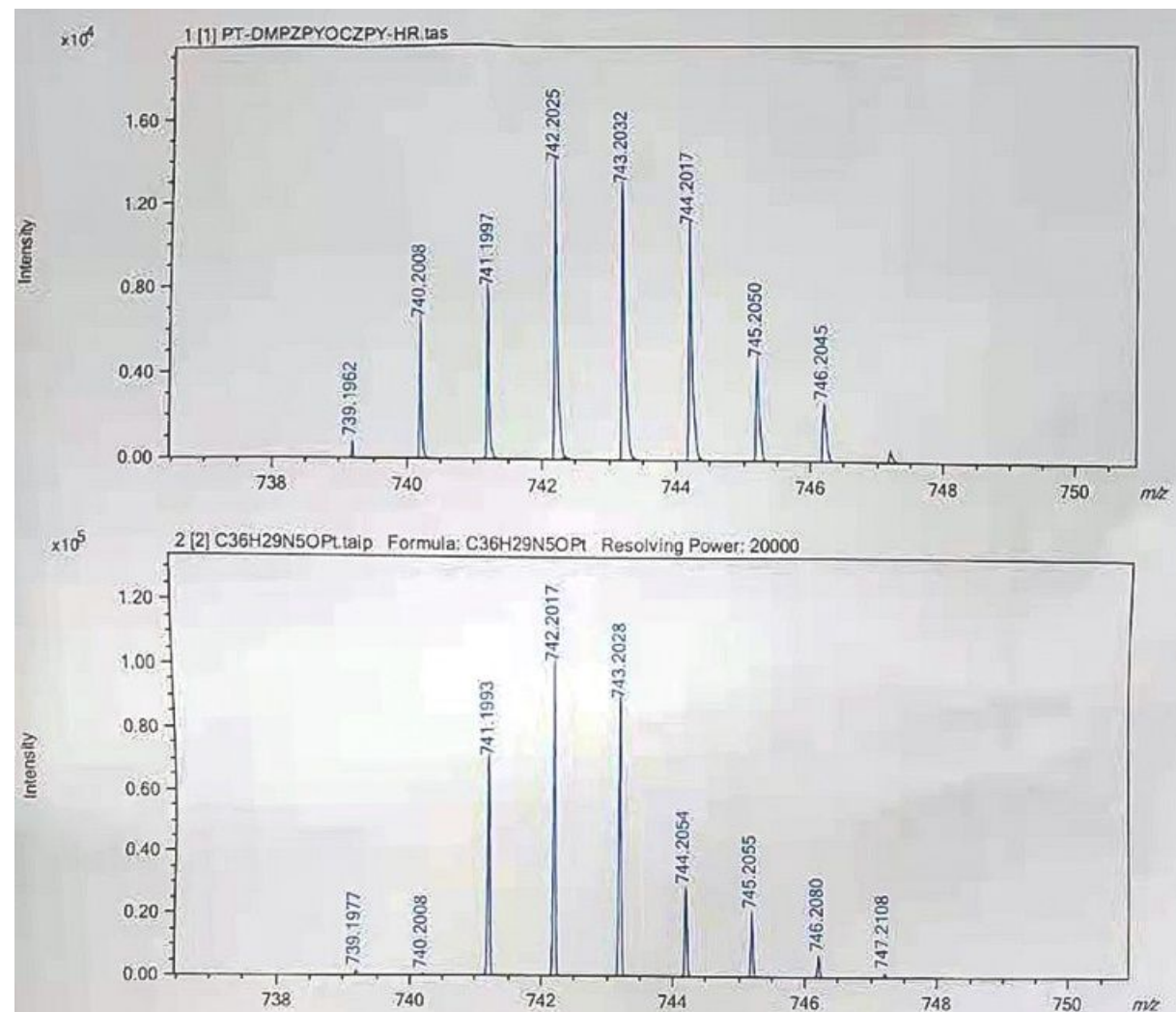

\begin{tabular}{|c|c|c|c|}
\hline $\mathrm{m} / \mathrm{z}$ & $\begin{array}{c}\text { Experimental } \\
\text { mass }\end{array}$ & Theoretical mass & $\begin{array}{c}\text { Relative } \\
\text { error(ppm) }\end{array}$ \\
\hline 739 & 739.1962 & 739.1977 & -2.03 \\
\hline 740 & 740.2008 & 740.2008 & 0.00 \\
\hline 741 & 741.1997 & 741.19934 & 0.49 \\
\hline 742 & 742.2025 & 742.20172 & 1.05 \\
\hline 743 & 743.2032 & 743.20283 & 0.50 \\
\hline 744 & 744.2017 & 744.2054 & -4.97 \\
\hline 745 & 745.205 & 745.20551 & -0.68 \\
\hline 746 & 746.2045 & 746.20799 & -4.68 \\
\hline
\end{tabular}

Figure S18 High resolution mass analysis of Pt(mpzpyOczpy-mesi) 